\title{
RECURRENT ORAL MUCOSITIS IN METASTATIC MELANOMA PATIENT TREATED WITH CHECKPOINT INHIBITOR, PEMBROLIZUMAB: CASE REPORT AND LITERATURE REVIEW
}

J. Dimitrijevic ${ }^{1}$, S. Bosnjak ${ }^{1}$ S. Matkovic², N. Babovic², S. Susnjar¹.

${ }^{1}$ Institute of Oncology and Radiology of Serbia, Oncology intensive care unit and supportive oncology mobile team, Belgrade, Serbia.

${ }^{2}$ Institute of Oncology and Radiology of Serbia, Department for soft tissue sarcoma- melanoma and urologic malignancies, Belgrade, Serbia

\section{Introductions}

Pembrolizumab is up to date strategy against various types of cancer including melanoma. It has an unique type of adverse events (AE's) described as immune-related AE's (irAE's) connected with excessive immune system activation. Immune-related oral mucositis (irOM) with pembrolizumab is rarely described in literature.

\section{Objectives}

To report a case of a 57-year-old patient with metastatic melanoma treated with pembrolizumab who developed a recurrent irOM

\section{Methods}

Assessment and treatment of irOM was performed according to the MASCC/ESMO guidelines for oral mucositis and ESMO guidelines for management of immunotherapy toxicity. IrAE's severity was assessed by CTCAE and WHO criteria. A retrospective literature search of published data was conducted.

\section{Results}

Patient developed grade 2 irOM after the first cycle and grade 4 irOM after the second cycle of pembrolizumab (Figure 1). OM was judged as an irAE's because of its recurrent nature and after other causes were excluded. IrOM of grade 4 was rather unresponsive to therapy: after one month it was downgraded to grade 3. Additional immunosuppressive agents are not accessible in Serbia for irAE's (off-label indication). Anti-PD1 antibody induced oral mucositis is reported, but rarely for pembrolizumab. Rapoport et al. 2017 described immunerelated oral manifestations in the form of lichenoid reactions, but not OM.
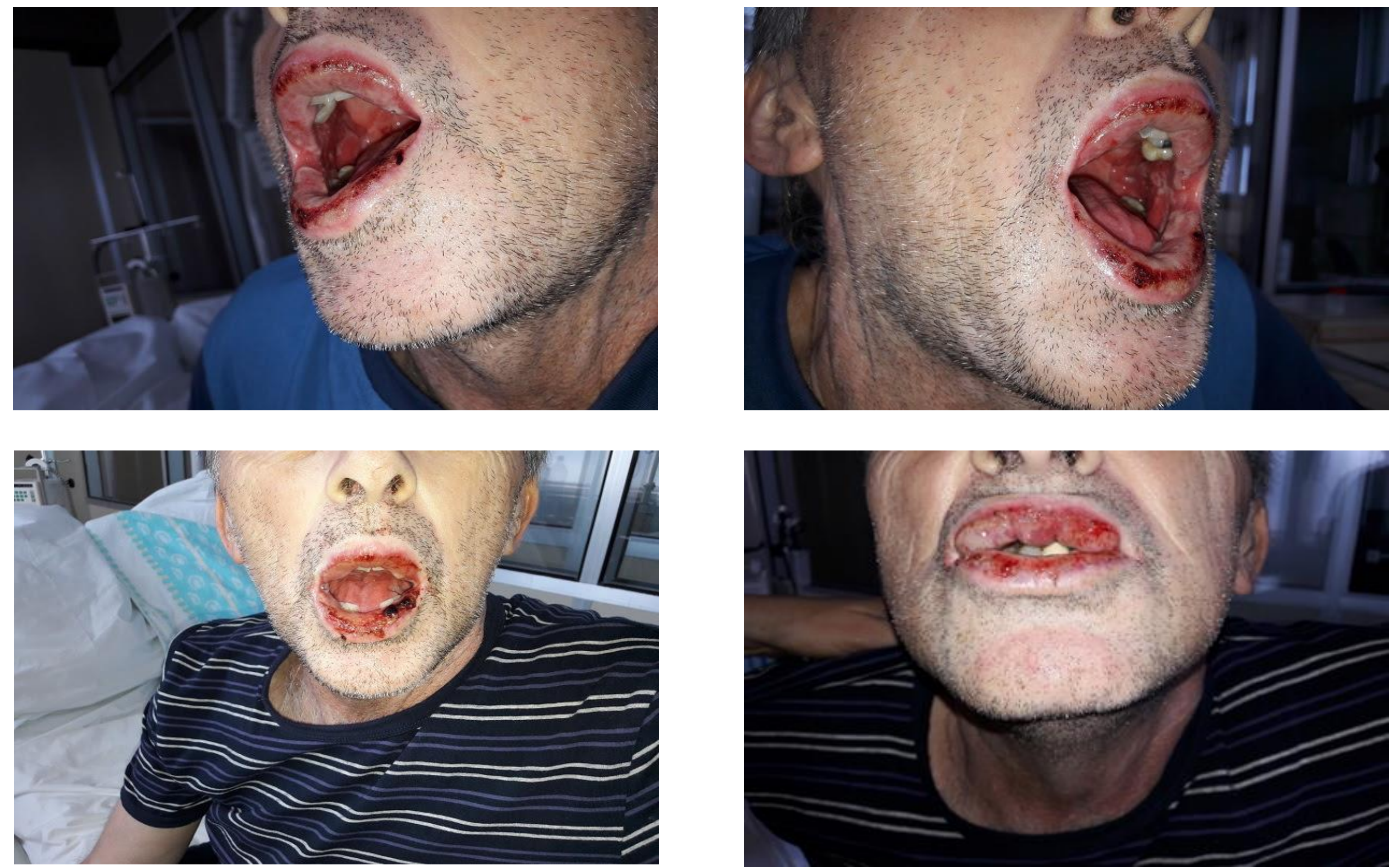

Fig. 1 Grade 4 irOM after the second cycle of pembrolizumab

* Methyprednisolone $1 \mathrm{mg} / \mathrm{kg}$ i.v
* Dexamethasone oral rinses
* Local analgesia (benzidamine oral solution and morphine oral solution
* Pystemic analgesia (fentanyl TD patch and i.v morphine)
* Parenteral antibiotic (cefazolin i.v)
Additional immunosupressives not accessible

\section{Conclusions}

Immune-related oral mucositis is an uncommon irAE of pembrolizumab. The best approach to the management of irOM is yet to be defined. 